\title{
Neue Möglichkeiten der ärztlichen Tätigkeit
}

\section{Jan von Overbeck}

Interessenbindung:

Der Autor ist Chief Medical Officer im Schweizer Zentrum für Telemedizin MEDGATE.

\section{* Die Literaturangaben finden sich unter www.saez.ch $\rightarrow$ Aktuelle Nummer oder $\rightarrow 2011 \rightarrow 45$.}

\section{Telearbeit}

Vor dem Hintergrund der Ölkrise hat sich in den 70er Jahren die Idee entwickelt, das physische Pendeln zum Arbeitsplatz zu ersetzen, indem Arbeitsvorgaben und Ergebnisse mit den Mitteln der Informationsund Kommunikationstechnologie über räumliche Distanz ausgetauscht werden. Es folgten diverse Modellversuche, vorwiegend innerhalb der IT-Branche, die aber vor allem durch recht hohe Kosten für die notwendige Infrastruktur behindert wurden [1]*. Heutzutage sind die dafür notwendigen Mittel der Informations- und Kommunikationstechnologie breit verfügbar, so dass sogenannte Telearbeitsmodelle immer häufiger umgesetzt werden [2]. Die vorhandenen Erdölreserven sind seit den 70er Jahren weiter geschrumpft, es sprechen jedoch, abgesehen von der Einsparung der für das Pendeln benötigten ökonomischen und ökologischen Ressourcen, noch andere Punkte für Telearbeit. Dem Arbeitnehmer ermöglicht Arbeiten von zu Hause aus eine ausgewogenere WorkLife-Balance und eine damit einhergehende grössere Zufriedenheit. Für die Unternehmen ist Telearbeit assoziiert mit höherer Produktivität, höherer Flexibilität und höherer Verfügbarkeit des Arbeitnehmers [3]. Die Unternehmen versprechen sich dadurch eine erhöhte Attraktivität als Arbeitgeber und überdies kann der Kreis der potenziellen Arbeitskräfte erweitert werden. Telearbeit wird aufgrund seiner Vorteile von den verschiedensten Kreisen propagiert, z. B. im Rahmen des Home Office Day, der insbesondere den Zuwachs an Lebensqualität, Produktivität und Klimaschutz in den Vordergrund stellt (www.homeofficeday.ch).

\section{Herausforderungen für Arbeitnehmer und Arbeitgeber}

Telearbeit stellt das Arbeitsverhältnis vor neue Herausforderungen. In erster Linie ist eine neue Vertrauensbasis zwischen Arbeitnehmer und Arbeitgeber notwendig. Dann müssen die organisatorischen, räumlichen, rechtlichen und technischen Fragen für den Arbeitsplatz zu Hause geklärt sein. Insbesondere müssen die Datensicherheit für die Arbeit zu Hause sowie der Transfer zum Arbeitgeber sichergestellt sein. Für den Arbeitnehmer kann die Abgrenzung zwischen Berufs- und Privatsphäre eine Herausforderung sein. Ausserdem kann Telearbeit zu einer geringeren sozialen Einbindung der Mitarbeiter und Identifikation mit dem Unternehmen führen [4]. Aus der Arbeitgeberperspektive stellen sich insbesondere Fragen rund um die Erreichbarkeit des Arbeitnehmers

\section{Nouvelles possibilités de travail pour les médecins}

Au cours de ces dernières décennies, le télétravail s'est progressivement établi. Etant donné qu'en télémédecine clinique, le médecin n'a par définition aucun contact physique avec le patient et que la consultation se déroule au moyen des technologies d'information et de communication, cette manière de fournir des prestations répond tout à fait aux modèles de télétravail. En télémédecine, le télétravail permet de fournir une prestation médicale clinique à distance tout en la conciliant avec des tâches familiales ou d'autres obligations.

und die Verzahnung mit anderen Arbeitsprozessen bzw. anderen Mitarbeitern.

\section{Ärztliche Telekonsultationen}

Mit der zunehmenden Verbreitung von Kommunikationstechnologien hat sich in der Schweiz zudem eine neue Form der Interaktion zwischen Arzt und Patient etabliert: die ärztliche Telekonsultation [5-7]. Die ärztlichen Konsultationen übers Telefon umfassen eine strukturierte Anamnese und Beratung des Patienten und können zu der Empfehlung einer Selbstbehandlung, einer physischen Arztkonsultation oder einer Spitaleinweisung im für die medizinische Situation jeweils adäquaten Zeitrahmen führen. Sie müssen den sogenannten «Best Practices» der Schweizerischen Gesellschaft für Telemedizin und eHealth (www.sgtm.ch) folgen, welche die juristischen Rahmenbedingungen sowie die Anforderungen an Qualität, fachliche Qualifikation des Personals, Infrastruktur und Serviceleistungen beschreiben. Dazu gehören eine mehrwöchige theoretische und praktische Einführungsphase aller neu eintretenden Ärztinnen und Ärzte. Dies liegt vor allem in der Tatsache begründet, dass bei der ärztlichen Arbeit über Distanz das Personal eingehend über die Möglichkeiten und Grenzen sowie die speziellen Anforderungen bei der Patientenbetreuung ohne physischen Kontakt geschult werden muss. Im Vordergrund steht hier das diagnostische Instrument der sorgfältig durchgeführten, struktu- 
1 Dostal W. Telearbeit in der Informationsgesellschaft. Göttingen: Verlag für angewandte Psychologie; 1999.

2 Grote G, Staffelbach B. Schweizer Human Relations Barometer 2010 - Arbeitsflexibilität und Familie. Zürich: Verlag Neue Zürcher Zeitung; 2010.

3 Stiftung Produktive Schweiz. Work Anywhere. Zwischen Produktivität und Lebensqualität: Herausforderungen und Chancen von flexiblen Arbeitsplatzmodellen. Zürich: Stiftung Produktive Schweiz; 2011.

4 Grote G, Manchen S, Springall L. Telemanagement: Notwendigkeit für ein komplexes Verhaltensrepertoire. Arbeit. 2004;13:48-60.

5 Agency for Healthcare Research and Quality (AHRQ). Telemedicine for the Medicare Population: Update. www. ahrq.gov/downloads/pub/evidence/pdf/telemedup/ telemedup.pdf

6 Rimner T, Blozik E, Begley C, Grandchamp C, von Overbeck J. Patient adherence to recommendations after teleconsultation: survey of patients from a telemedicine centre in Switzerland. J Telemed Telecare. 2011;17:235-9.
7 Blozik E, Sommer-Meyer C, Cerezo M, von Overbeck J. Effectiveness and safety of telemedical management in uncomplicated urinary tract infections. J Telemed Telecare. 2011;17:78-82.

8 Von Overbeck J, Blozik E. Replik und Duplik auf das Editorial «Notfallanruf: Grenze und Gefahren» Praxis (Bern 1994). 2010;99:1451-2.

9 The American Telemedicine Association. Core Standards for Telemedicine Operations. www.americantelemed.org/files/public/standards/Core Standards_withCOVER.pdf

10 Kraft E. 30273 Ärztinnen und Ärzte für die Schweiz. Schweiz Ärztezeitung. 2011;92:440-4.

11 Kraft E, Hersperger M. Ärzteschaft in der Schweiz die Feminisierung der Medizin. Schweiz Ärztezeitung. 2009; 90:1823-5.

12 Faller A, Kaufmann S. Telemedizin: Fachwissen weltweit vermitteln. Managed Care. 2007;6:17-20. 